\title{
Heart rate variability in the frequency domain after strength training with citrus aurantium supplementation
}

\begin{abstract}
Objective:The objective of the study was to verify the autonomic response by analyzing heart rate variability in the frequency domain, after strength training with supplementation of citrus aurantium.

Method: Participated in the present study, 10 men $(28.2 \pm 3.8$ years; $1.760 .03 \mathrm{~m} ; 79.33 .9 \mathrm{~kg}$; 25.52.1 BMI) trained with previous experience in strength training activity of at least six months. Heart rate variability (HRV) was measured in a 5-minute window over 10 minutes with the individual in a sitting position, before, in two pre-exercise moments (before and 30 minutes after supplementation), immediately after exercise for 60 minutes. Frequency domain indices were used for HRV analysis, being: The components of low frequency (LF - sympathetic activation indicator), high frequency (HF - parasympathetic activation indicator) and the ratio between low and high frequency ( $\mathrm{LF} / \mathrm{HF}$ - sympathetic vagal balance indicator). Participants supplemented $975 \mathrm{mg}$ of citrus aurantium dry extract (6\% synephrine) (S) or maltodextrin (P) placebo. They performed five sets with loads of $70 \%$ 1-RM, in the horizontal bench press exercise $(\mathrm{SH})$ with supplementation with citrus aurantium or with the intake of placebo. The Shapiro-Wilk normality test was used and a repeated-measures ANOVA was conducted to analyze all samples, cardiovascular, followed by a Fisher post hoc, when necessary. All analyzes were performed using SPSS version 21.0 (IBM, I.C.). The significance value adopted was $\mathrm{p} \leq 0.05$ in all tests.
\end{abstract}

Results: For LF, there were no significant differences between the supplementation conditions ( $p=0.063$ ), as well as the different supplementation conditions, where no significant changes were observed $(p=0.177)$ and for the different moments of checks $(p=0.085)$. In the HF, there were significant differences between the interactions $(p=0.043)$, however, for the two conditions, supplementation $(\mathrm{p}=0.317)$ and different verification moments $(\mathrm{p}=0.178)$, no significant differences were verified. In LF/HF there were significant differences in the interaction ( $p=0.011)$ and between the different supplementation conditions, there were no significant changes $(\mathrm{p}=0.626)$. Between the moments of verification, differences were also observed $(\mathrm{p}=0.032)$.

Conclusion: The present study did not find significant differences in heart rate variability with different types of supplementation. However, supplementation with citrus aurantium was able to stimulate the autonomic sympathetic response even at the pre-exercise moment, signaling that p-synephrine may present some changes in the cardiovascular system. Thus, it is worth paying attention to the prescriptions of this supplement.

Keywords: heart rate variability, strength training, citrus aurantium
Volume 5 Issue 3 - 2020

\author{
Leandro de Oliveira Sant'Ana, ${ }^{1,2,3}$ Matheus \\ José Lima, ${ }^{4}$ Renata Simoni, ${ }^{5}$ Estevão \\ Scudese, ${ }^{3}$ Cristiano Queiroz de Oliveira, ${ }^{3,4}$ \\ Fabiana Rodrigues Scartoni, ${ }^{3,4}$ Gilmar Weber \\ Senna ${ }^{3,4}$ \\ 'Post Graduate Program in Physical Education, Federal \\ University of Juiz de Fora, Brazil \\ ${ }^{2}$ Strength Training Studies and Research Laboratory, Federal \\ University of Juiz de Fora, Brazil \\ Laboratory of Sport and Exercise Sciences, Catholic University \\ of Petrópolis, Brazil \\ ${ }^{4}$ Department of Physical Education, Catholic University of \\ Petrópolis, Brazil \\ ${ }^{5}$ Department of Nutrition, Arthur Sá Earp Neto College, Brazil
}

Correspondence: Leandro de Oliveira Sant'Ana, Faculty of Physical Education and Sports, UFJF

losé Lourenço Kelmer Street, S/N - University Campus, São

Pedro, Brazil, Email losantana.ufjf@gmail.com

Received: May 02, 2020 | Published: May II, 2020

\section{Introduction}

Heart rate variability (HRV) is a physiological parameter of interest to behavioral and biomedical scientists. ${ }^{1}$ In the clinical setting, HRV metrics are often used to stratify cardiovascular risk. ${ }^{2}$ Likewise, HRV has been used in the field of sport, to control internal load ${ }^{3-4}$ and as a biomarker of physiological responses related to nutrition in the potential for improving health and performance. ${ }^{5}$ However, studies with HRV analysis in different interventions and manipulations of exercises and ergogenic resources have been elaborated to better understand the importance of this variable in health and performance.

Synephrine, also known as p-synephrine, consists of an alkaloid with an adrenergic activity that is naturally found in bitter oranges and other citrus fruits and can be added as the active ingredient in sports supplements, in the form of dry extract of bitter orange (citrus aurantium), to promote weight loss, improve athletic performance and increasing energy, without causing adverse effects on the cardiovascular system. ${ }^{6,7}$ Synephrine has been frequently used in "pre-workout" supplements, which have thermogenic properties, as a substitute for ephedrine, since it has been banned from commercialization due to its strong association with serious cardiovascular effects. ${ }^{8}$

Strength training is a physical exercise recommended by the main normative agency of physical activity (American College of Sports Medicine), as an activity of relative safety, which provides health benefits. ${ }^{9}$ In the training scenario, mainly of strength, the use of supplements presents an increasing increase among athletes and individuals practicing physical activity with different goals. ${ }^{10}$ Thus, due to the scarcity of studies examining the effects of isolated p-synephrine in training, the objective of the present study was to verify the autonomic response with HRV analysis in the frequency domain. 


\section{Material \& methods}

\section{Participants}

Participated in this study, 10 men trained with previous experience in strength training activity for at least six months (Table 1). The inclusion criteria were: a) having a medical indication and/or clearance to perform physical exercises; b) who answered previously and negatively all questions in the physical activity readiness questionnaire (PAR-Q); c) not having any acute or chronic health condition that could compromise the performance of physical activities; d) did not use drugs to treat pre-established endocrine diseases, heart disease, high blood pressure, asthma or any musculoskeletal conditions that could serve as an intervening factor in the practice of the activity; e) did not have neurological disorders and used drugs that could cause attention disorders. This study met the standards for researching human beings, by Resolution 466 of 2012, of the National Health Council of 07/04/2016 and the Declaration of Helsinki. The project was approved by the Research Ethics Committee of the Arthur Sá Earp Neto Faculty for ethical assessment, under protocol 2,586,986 and CAAE 80475417.0.0000.5245.

Table I Characterization of the sample population

\begin{tabular}{lll}
\hline Sample & $\begin{array}{l}\text { Mean } \begin{array}{l}\text { deviation } \\
\text { Age }(\text { years })\end{array} \\
28,2 \pm 3,8\end{array}$ & Min - Max \\
\hline Statures $(\mathrm{m})$ & $1,76 \pm 0,03$ & $1,7 \mathrm{I}-\mathrm{I}, 80$ \\
Weight $(\mathrm{kg})$ & $79,3 \pm 3,9$ & $74, \mathrm{I}-85,0$ \\
BMI $\left(\mathrm{kg} / \mathrm{m}^{2}\right)$ & $25,5 \pm 2,1$ & $23,3-29,0$ \\
Waist circumference $(\mathrm{cm})$ & $84,9 \pm 3,3$ & $82,1-90$ \\
$\%$ fat $(3)$ & $13,6 \pm 3,4$ & $10,8-19,4$ \\
$\%$ fat $(7)$ & $15,3 \pm 3,3$ & $12,0-19,3$ \\
Load test I RM $(\mathrm{kg})$ & $88,4 \pm 10,4$ & $72-98$ \\
Relative strength $\left(\mathrm{kg} / \mathrm{m}^{2}\right)$ & $1,12 \pm 0,17$ & $0,88-1,32$ \\
\hline
\end{tabular}

\section{Analysis of heart rate variability}

HRV analysis was measured in a 5-minute window over a 10-minute period with the individual in a sitting position. HRV was measured before in two moments before exercise (before and 30 minutes after supplementation), immediately after exercise and for 60 minutes after the end of the intervention in an interval of 10 in 10 minutes. A POLAR RS800CX ${ }^{\circledR}$ watch (Multisport, Kempele, Finland) TM was used to collect HRV. ${ }^{11}$ For HRV treatment, the data was transferred to the computer and attached to Polar Trainer 5 Software ${ }^{\circledR}$. Correction procedures for all data were performed on this platform and subsequently filed in TXT format for the start of treatment in Kubios HRV Standart Software ${ }^{\circledR}$, version 3.3.1. Then, the values resulting from the frequency domain were noted for the low-frequency $(\mathrm{LF} ; 0.04-0.15 \mathrm{~Hz})$, high-frequency $(\mathrm{HF} ; 0.16-0.4 \mathrm{~Hz})$ data and the $\mathrm{LF} / \mathrm{HF},{ }^{12}$ corresponding indicators of sympathetic, parasympathetic activation and vagal sympathetic balance, respectively.

\section{Control of food consumption}

All participants received a form prepared by the researcher, containing instructions for filling out the food register. The records were filled out by each individual, in which they described their food consumption of solids and liquids in the 24 hours before the tests, to verify possible nutritional interferences that could benefit their performance in the tests. The analysis of food consumption was made qualitatively, to verify possible food interference in the tested supplementation.

Additionally, the participants received standardized foods one hour before performing the experimental procedures, to guarantee the same dietary conditions for each individual and allow a favorable condition for the analysis. The snacks were designed to provide recommended amounts of carbohydrate and protein to the patient. the practice of physical activity. To ensure a balanced state of hydration during exercise, each individual was instructed to drink 5 to 10 milliliters of water per kilogram of body weight, 2 hours before the analysis sessions. ${ }^{13}$ The meals offered contained an average of $278.8 \mathrm{kcal}$, being: 45.32 .2 grams of carbohydrate; 9.10 .4 grams of protein; 6.8 $0.3 \mathrm{~g}$ of lipids and 5.70 .3 grams of fiber per meal offered. Regarding hydration, the average water offered to each participant was 594.6 29.2 milliliters. $^{14}$

\section{Study design}

Visits for data collection took place one week apart, the first being one week after the date of the 1-RM test. Upon arriving at the study site, the participants were placed in a sitting position for 10 minutes. After at least 10 minutes of sitting rest, the participants underwent HRV checks. After 5 minutes, the participants were randomly selected and randomly, in a counterbalanced and doubleblind manner, supplemented with $975 \mathrm{mg}$ of the dry extract of citrus aurantium ( $6 \%$ synephrine) $(\mathrm{S})$ or placebo of maltodextrin $(\mathrm{P})$. About 30 min after taking the supplements, HRV was checked again. Only after this procedure, the participants started the exercise sessions. The experiment was repeated using the alternative supplement managed in a balanced way after a week of washout between sessions. Four days (96h) after the 1-RM test, the participants were alternately ordered to carry out two separate visits for a week, consisting of five series with loads of $70 \% 1-\mathrm{RM}$, for the bench press exercise. horizontal with citrus aurantium supplementation or placebo ingestion.

For both sessions, the subjects performed a previous warm-up, which consisted of two sets of 12 repetitions with 40\% 10-RM. An interval of two minutes was respected after the warm-up and before the series. ${ }^{15}$ The repetition speeds were auto-selected (with no attempt to control). ${ }^{16}$ The effort perceived through the OMNI scale ${ }^{17}$ was recorded after the execution of each series. All visits were conducted between 8:00 am and 10:00 am. This time was selected to avoid, as much as possible, the cumulative effects of the course of the day in the circadian cycle of the participants. ${ }^{18}$

\section{Statistical analysis}

All metric scale data were presented according to their mean and standard deviation (Mean SD). After all analyzes, the variables show normal distribution (Shapiro-Wilk normality test), so a repeated-measures ANOVA was conducted to analyze all samples, cardiovascular, followed by a Fisher post hoc, when necessary. All analyzes were performed using SPSS version 21.0 (IBM, I.C.). The significance level adopted was $\mathrm{p} \leq 0.05$ in all tests (Figure 1).

For HF (parasympathetic activation), significant differences were observed between the interactions $(\mathrm{p}=0.043)$, however, for the two conditions, supplementation $(\mathrm{p}=0.317)$ and different verification moments $(\mathrm{p}=0.178)$, were not verified significant differences (Figure 2). 


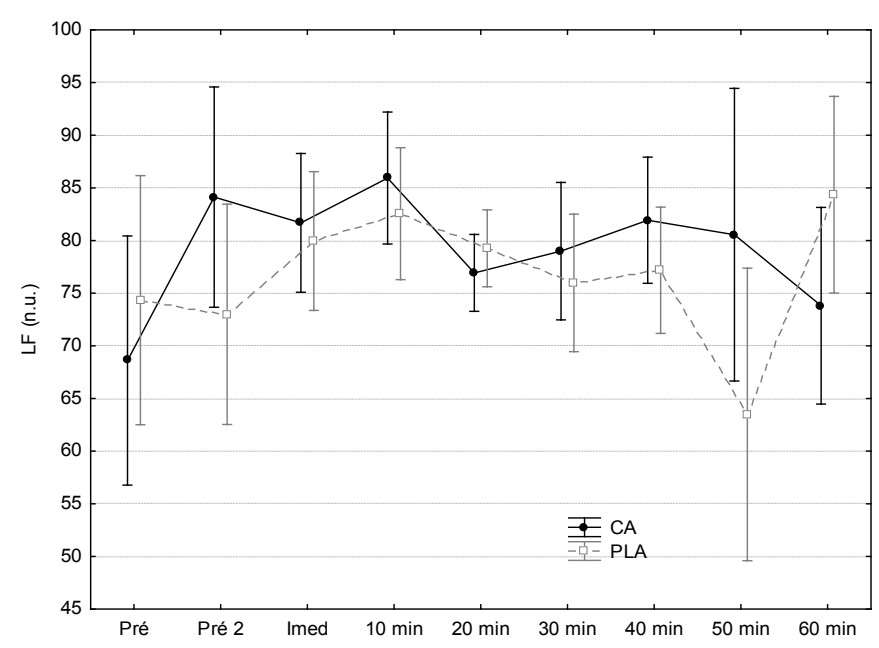

Figure I Low frequency in both supplementation conditions.

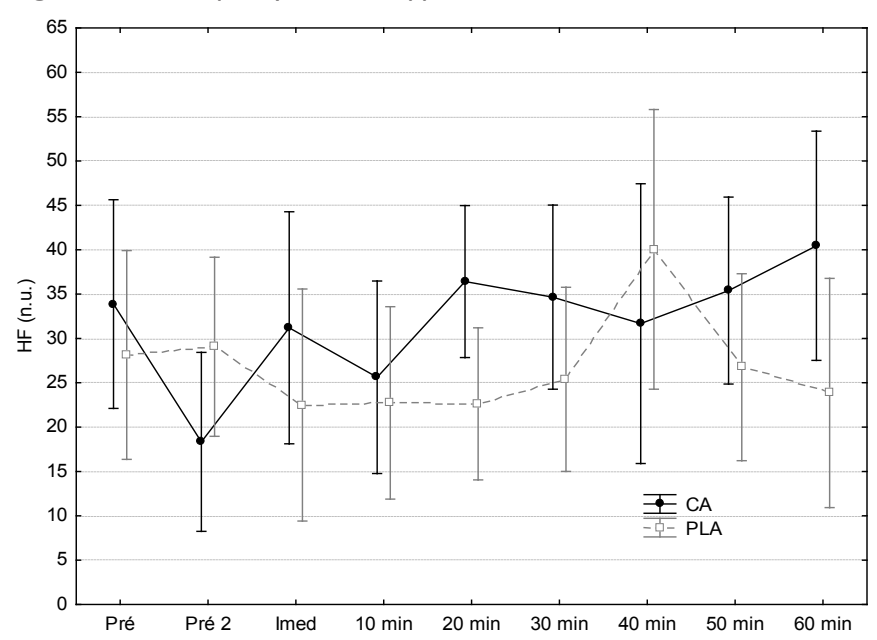

Figure 2 High frequency in both supplementation conditions.

About the sympathetic-vagal relationship, significant differences for the interaction were observed $(\mathrm{p}=0.011)$. It is worth mentioning, however, that among the different supplementation conditions, there were no significant changes $(p=0.626)$. Between the moments of verification, differences were observed $(\mathrm{p}=0.032)$, showing an important increase in sympathetic activation as the use of the supplement and 10 minutes after exercise (Figure 3).

\section{Discussion}

The objective of the present study was to verify the behavior of HRV, in the frequency domain, after strength training with the supplementation of citrus aurantium. In our findings, there was greater activation of the sympathetic autonomic system with a reduction in parasympathetic activity in the condition of citrus aurantium. Thus, when analyzing the sympathetic-vagal relationship, we found that only for the citrus aurantium condition the differences were significant, from the moment of pre-exercise concerning the pre-initial increase. This indicates that supplementation with citrus aurantium was able to stimulate the autonomic sympathetic response even at the pre-exercise moment, signaling that, unlike what has already been described in the literature, $\mathrm{p}$-synephrine may present some changes in the cardiovascular system. Therefore, it is suggested restrictions for the indication of this supplement for individuals who have some type of associated disease, corroborating what was warned by the Comprehensive Database of Natural Medicines. ${ }^{19}$

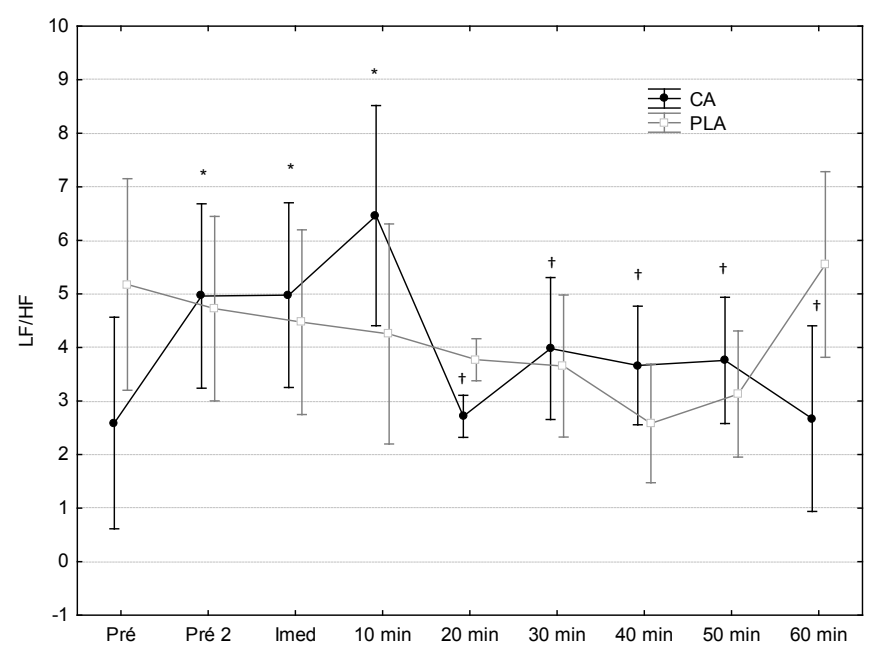

Figure 3 Low and high frequency ratio in both supplementation conditions.

*Significant differences for pre-initial verification. $\nmid$ significant differences for the 10 min check.

It is known that cardiovascular effects are associated with mechanisms of binding to adrenergic receptors. For men and animals, adverse cardiovascular effects are not commonly associated with p-synephrine, although such effects are widely known when associated with the consumption of ephedrine and ephedra. ${ }^{20}$ The effects on HRV with the use of p-synephrine are believed to occur due to the poor binding of $p$-synephrine to $\alpha-1, \alpha-2$, and $\beta-1$, and $\beta-2$ adrenergic receptors when compared to other ligands such as norepinephrine, epinephrine, ephedrine, and m-synephrine. ${ }^{21}$ However, as previously described, $\mathrm{p}$-synephrine is linked, albeit at a low level, to $\alpha$ and $\beta$ adrenoreceptors, and some cardiovascular events may occur through these receptors, ${ }^{22}$ as appears to have occurred in the present study.

Specifically, Ratamess et $\mathrm{al}^{23}$ in an experiment evaluated 12 participants with at least 6 months of training. In that study, the condition called synephrine (S) received $100 \mathrm{mg}$ of synephrine; the SCF condition received $100 \mathrm{mg}$ of caffeine $+100 \mathrm{mg}$ of synephrine and the placebo condition $(\mathrm{P})$ received the placebo (maltodextrin in negligible amounts). Ratamess et al..$^{23}$ observed significant differences $(p=0.05)$ between treatments when the total number of repetitions was analyzed. Precisely, the SFC and S groups produced significantly more repetitions than P. However, there was no significant effect between the different supplements when verifying the number of repetitions in each series.

Also, Jung et al. ${ }^{24}$ observed 25 healthy male and female participants who ingested a $2 \mathrm{~g}$ maltodextrin (PLA) placebo; a preworkout containing beta-alanine $(3 \mathrm{~g})$, creatine nitrate $(2 \mathrm{~g})$, arginine alpha-ketoglutarate (2 g), N-acetyl-L-tyrosine (300 mg), caffeine (284 mg), Mucuna pruiriens standardized extract for 15\% 1-dopa (15 $\mathrm{mg}$ ), vitamin $\mathrm{C}$, ascorbic acid $(500 \mathrm{mg})$, niacin $(60 \mathrm{mg})$, folic acid $(50 \mathrm{mg})$ and vitamin B12 (70 $\mathrm{mg})$ and $2 \mathrm{~g}$ of maltodextrin (PWS); or PWS added citrus aurantium extract (PWS $+\mathrm{S}$ ) standardized for $30 \%$ p-synephrine $(20 \mathrm{mg})$. There was no statistically significant difference between the types of supplementation concerning the total 
volume of repetitions for horizontal bench press and leg press or in the performance of the sprints. The present study corroborates with Ratamess et al..$^{23}$ considering that it was found that the participants who ingested citrus aurantium performed a greater total number of repetitions compared to those who consumed the placebo. The present study is composed of some limitations. The number of participants was small, therefore, it is suggested new studies with a larger number of participants with the same analysis objectives. However, further investigation into the effects of citrus aurantium on the cardiovascular system will be needed. Investigate other variables in this system, as well as other HRV indices, such as the time domain.

\section{Conclusion}

The present study did not find significant differences in heart rate variability with different types of supplementation. The main response was to change the vagal sympathetic difference where there were no significant changes with citrus aurantium. However, supplementation with citrus aurantium was able to stimulate the autonomic sympathetic response even at the pre-exercise moment, signaling that $\mathrm{p}$-synephrine may present some changes in the cardiovascular system. Thus, it is worth paying attention to the prescriptions of this supplement, especially for individuals with a history of cardiovascular disorder.

\section{Funding}

None.

\section{Acknowledgements}

Universidade Católica de Petrópolis, Rio de Janeiro, Brasil.

Universidade Federal de Juiz de Fora, Minas Gerais, Brasil.

\section{Conflicts of interest}

The authors declare no conflicts of interest.

\section{References}

1. de Geus EJC, Gianaros PJ, Brindle RC, et al. Should heart rate variability be "corrected" for heart rate? Biological, quantitative, and interpretive considerations. Psychophysiology. 2019; 56(2): e13287.

2. Laborde S, Mosley E, Thayer JF, et al. Heart rate variability and cardiac vagal tone in psychophysiological research-recommendations for experiment planning, data analysis, and data reporting. Frontiers in Psychology. 2017;8:213.

3. Nakamura FY, Flatt AA, Pereira LA, et al. Ultra-short-term heart rate variability is sensitive to training effects in team sports players. Journal of Sports Science and Medicine. 2015;14(3):602-605.

4. Salahuddin N, Shafquat A, Marashly Q, et al. Increases in heart rate variability signal improved outcomes in rapid response team consultations: A cohort study. Cardiology Research and Practice. 2018;1590217:1-8.

5. Young HA, Benton D. Heart-rate variability: a biomarker to study the influence of nutrition on physiological and psychological health? Behavioural Pharmacology. 2018;29(2):140.

6. Rossato LG1, Costa VM, Limberger RP, et al. Synephrine: from trace concentrations to massive consumption in weight-loss. Food Chemical and Toxicology. 2011;49(1):8-16.

7. Hansen KD, George NI, White GE, et al. Physiological effects following administration of Citrus Aurantium for 28 days in rats. Toxicology Applied Pharmacology. 2012;261(3):236-247.
8. Bent S1, Padula A, Neuhaus J. A review of the safety and efficacy of Citrus Aurantium in weight management. Obesity: Epidemiology, Pathophysiology, and Prevention. 2007;371-382.

9. American college of sports medicine. position stand: progression models in resistance training for healthy adults. Medicine Science Sports Exercise. 2002;34(2):364-380.

10. Lopez HL, Habowski SM, Weir JP, et al. Eight weeks of supplementation with a multi-ingredient weight loss product enhances body composition, reduces hip and waist girth, and increases energy levels in overweight men and women. Journal of the International Society of Sports Nutrition. 2013;10(22):1-14.

11. Barbosa MP, da Silva NT, de Azevedo FM, et al. Comparison of Polar $^{\circledR}$ RS800G3 ${ }^{\mathrm{TM}}$ heart rate monitor with Polar $^{\circledR}$ S810i ${ }^{\mathrm{TM}}$ and electrocardiogram to obtain the series of RR intervals and analysis of heart rate varibility at rest. Clin Physiol Funct Imagining. 2016;36:112-117.

12. Task Force. Heart rate variability: standards of measurement, physiological interpretation and clinical use. Task force of the european society of cardiology and the north american society of pacing and electrophysiology. Circulation 1996;93(5):1043-1065.

13. American College of Sports Medicine. joint position statement: nutrition and athletic performance. Medicine Science Sports Exercise. 2016;48(3):543-568.

14. Scudese E, Simo R, Seena G, et al. Long rest interval promotes durable testosterone responses in high-intensity bench press. The Journal of Strength \& Conditioning Research. 2016;30(5):1275-1286.

15. Senna. Rest period length manipulation on repetition consistency for distinct single-joint exercises. Journal of Exercise Physiology, 2016;19(5):90-101.

16. Senna G, Salles BF, Prestes J, et al. Influence of two different rest interval lengths in resistance training sessions for upper and lower body. Journal of Sports Science and medicine. 2009;8(2):197-202.

17. Lagally KM, Robertson RJ. Construct validity of the OMNI resistance exercise scale. Journal of Strength and Conditioning Research. 2006;20(2):252-256.

18. Scudese E. The effect of rest interval length on repetition consistency and perceived exertion during near maximal loaded bench press sets. Journal of Strength and Conditioning Research, 2015;29(11):30793083.

19. Natural medicines comprehensive database. Bitter orange, 2017.

20. Haller CA, Benowitz NL. Adverse cardiovascular and central nervous system events associated with dietary supplements containing ephedra alkaloids. The New England Journal of Medicine. 2000;343(25):18331838 .

21. Stohs SJ, Badmaev V. A review of natural stimulant and non-stimulant thermogenic agents. Phytotherapy Reserch. 2016;(5):732-740.

22. Shara M, Stohs SJ, Mukattash TL, et al. Cardiovascular safety of oral p-synephrine (bitter orange) in human subjects: a randomized placebo-controlled cross-over clinical trial. Phytotherapy Research. 2016;30(5):842-847.

23. Ratamess NA, Bush JA, Kang J, et al. The effects of supplementation with P-Synephrine alone and in combination with caffeine on resistance exercise performance. Journal of the International Society of Sports Nutrition. 2015;12(35):1-11.

24. Y Peter J, Conrad PE, Majid K. Effects on ingesting a pre-workout dietary supplement with and without synephrine for 8 weeks on training adaptations in resistence-trained males. Journal of the International Society of Sports Nutrition. 2017;14(1):1-18. 